\title{
Challenges and perspective of drug repurposing strategies in early phase clinical trials
}

\author{
Shumei Kato ${ }^{1}$, Stacy L. Moulder ${ }^{2}$, Naoto T. Ueno${ }^{2}$, Jennifer J. Wheler ${ }^{1}$, Funda Meric- \\ Bernstam ${ }^{1}$, Razelle Kurzrock ${ }^{3}$, Filip Janku ${ }^{1}$ \\ ${ }^{1}$ Department of Investigational Cancer Therapeutics, The University of Texas MD Anderson Cancer Center, Houston, Texas, \\ USA \\ 2 Department of Breast Medical Oncology, The University of Texas MD Anderson Cancer Center, Houston, Texas, USA \\ ${ }^{3}$ Center for Personalized Cancer Therapy and Division of Hematology and Oncology, Department of Medicine, UC San Diego \\ Moores Cancer Center, La Jolla, California, USA
}

Correspondence to: Filip Janku, email: FJanku@mdanderson.org

Keywords: Drug repurposing, early phase trial, cancer, drug development

Received: June 16,2015 Accepted: June 28, $2015 \quad$ Published: June 30, 2015

This is an open-access article distributed under the terms of the Creative Commons Attribution License, which permits unrestricted use, distribution, and reproduction in any medium, provided the original author and source are credited.

\section{ABSTRACT}

Despite significant investments in the development of new agents only $5 \%$ of cancer drugs entering Phase I clinical trials are ultimately approved for routine clinical cancer care. Drug repurposing strategies using novel combinations of previously tested anticancer agents could reduce the cost and improve treatment outcomes. At MD Anderson Cancer Center, early phase clinical trials with drug repurposing strategies demonstrated promising outcomes in patients with both rare and common treatment refractory advanced cancers. Despite clinical efficacy advancing drug repurposing strategies in the clinical trial trajectory beyond early phase studies has been challenging mainly due to lack of funding and interest from the pharmaceutical industry. In this review, we delineate our experience and challenges with drug repurposing strategies.

\section{INTRODUCTION}

Despite the tremendous resources invested in anticancer therapy, cancer remains among leading causes of mortality worldwide [1]. Only 5\% of oncology drugs entering Phase I clinical trials are ultimately approved, and drug development takes an average of 13 years at a cost as high as $\$ 1.8$ billion [2]. The prolonged duration and enormous costs of the clinical trials required for regulatory approval by the U.S. Food and Drug Administration (FDA) emphasize the need for alternative strategies.

Relatively little attention is paid to utilizing existing drugs in novel combinations and regimens for enduring cancer indications. The major advantages of this "drug repurposing" approach are that the preclinical, pharmacokinetic, pharmacodynamic, and toxicity profiles of the drugs are already known and thus the repurposed regimens may rapidly translate into Phase II/III clinical studies. Drug repurposing also could reduce the costs of developing new drug therapies, especially when the patent protection expires, allowing generic manufacturing.
Although drug repurposing and development of new combinations using existing agents are getting more attention [2], and multiple studies have shown their potential benefit in cancer care [3-8], the design, conduct, and most importantly funding of such studies remain major challenges. Here we discuss our experience with this approach in the Department of Investigational Cancer Therapeutics (Phase I Clinical Trials Program) at The University of Texas MD Anderson Cancer Center and the challenges facing the broader application of this approach.

Drug repurposing strategies for cancer therapy in early phase clinical trials

Our department has been conducting broad early phase studies across disease types and molecular targets for the past 10 years. We have developed multiple clinical trials using a variety of repurposing strategies, and some of the approaches have resulted in very encouraging data. These trials were supported almost exclusively by 
institutional funds, and despite very promising results that often surpassed outcomes for emerging experimental anticancer agents, the lack of funding and other support have precluded further development. Some of these combinations are described here.

A Phase I clinical trial combining liposomal doxorubicin, bevacizumab, and temsirolimus (DAT) for patients with advanced cancers was designed to test the preclinical rationale that resistance to anthracyclines is driven through upregulation of hypoxia-inducible factor alpha (HIF-1 $\alpha)$, which promotes angiogenesis and tumor survival. Thus inhibiting angiogenesis, such as with the VEGF inhibitor bevacizumab, may overcome anthracycline resistance. However, resistance to bevacizumab is also driven by upregulation of HIF- $1 \alpha$. Addition of temsirolimus, a potent inhibitor of mTOR and consequently HIF-1 $\alpha$, can overcome this resistance [9]. During the dose-escalation phase we noticed remarkable activity in several distinct tumor types, including metaplastic breast cancer (MpBC) [8] and gynecologic malignancies [6].

$\mathrm{MpBC}$ is a rare subtype of breast cancer that typically does not express estrogen/progesterone receptors or human epidermal growth factor receptor 2 (HER2) and thus usually is treated as a triple-negative breast cancer. However, $\mathrm{MpBC}$ patients experience more disease recurrence, and associated poorer overall survival, than other patients with triple-negative breast cancer and generally have a poor response to systemic therapy [10]. Therefore there is no standard therapy for $\mathrm{MpBC}$. Despite the aggressive nature of this cancer, we observed 2 responses ( 1 complete [CR] and 1 partial response [PR]) in the 5 patients with treatment-refractory $\mathrm{MpBC}$ who received the DAT regimen on this trial. The $\mathrm{CR}$ was seen after 6 cycles of DAT, and the patient has been cancer free for more than 5 years and continues on maintenance therapy with the mTOR inhibitor as a single agent [8]. Encouraged by the promising beginning, we extended this cohort and observed objective responses in 5 of the 12 patients with $\mathrm{MpBC}(42 \%, 2 \mathrm{CR}$ and $3 \mathrm{PR})$ and stable disease $(\mathrm{SD})>6$ months in another 6 [50\%] [6].

The DAT regimen was effective in diverse cancer types, including endometrial endometrioid carcinoma (6/15 [40\%] PR), epithelial ovarian carcinoma (4/23 $[17 \%] \mathrm{PR})$, and parotid gland adenocarcinoma (4/6 [67\%] PR) [6]. On the basis of these promising outcomes, we have approached a cooperative group about conducting further clinical trials of the DAT regimen for $\mathrm{MpBC}$. However, as of this writing, the study has not been opened. This contrasts starkly with the rapid approval of crizotinib for anaplastic lymphoma kinase gene-rearranged nonsmall cell lung cancer (NSCLC); crizotinib was approved approximately 2 years after its anticancer activity was initially observed [11].

Another example of an early phase clinical trial using a repurposing approach is our Phase I experience

\section{Selected FDA approved targeted therapies and Phase I repurposing strategies from MDACC}

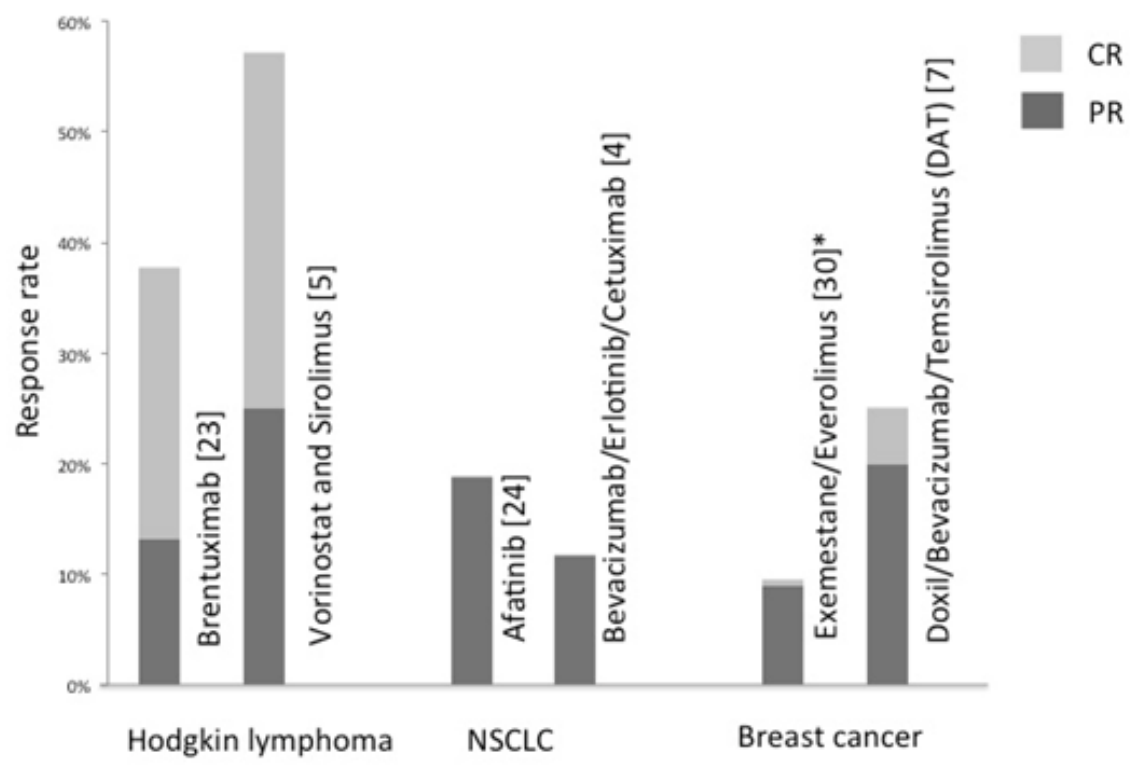

Figure 1: Overall response rate of selected FDA-approved novel targeted therapies (left) and repurposing-based combinations (right) in early phase clinical trials. Numbers in parentheses are the citation of the clinical trial. All response rates shown are from Phase I trials, except * from Phase III trial data [30]. 
with the combination of mTOR inhibitor sirolimus and vorinostat in heavily pretreated patients with advanced cancer. Vorinostat is a histone deacetylase (HDAC) inhibitor that is approved by the FDA for treatment of cutaneous T-cell lymphoma and has a single-agent response rate of 4\% in Hodgkin lymphoma refractory to standard therapies [12]. Novel HDAC inhibitors panobinostat or mocetinostat demonstrated objective response rates of $27 \%$ in the same patient population [13, 14]. Preclinical studies suggested that HDAC inhibitors, besides increasing histone acetylation, reduce the activity of AKT; however, that is circumvented by increasing mTOR activation through inhibition of LKB1 and adenine monophosphate-activated protein kinase. In preclinical studies, HDAC inhibitors were able to overcome mTORinduced resistance [15], and this synergistic activity became the rationale for our Phase I clinical trial of sirolimus and vorinostat.

During the dose-escalation phase, we observed anticancer activity in hepatocellular carcinoma and perivascular epithelioid cell tumor (data not shown) and most notably in treatment-refractory Hodgkin lymphoma. The 28 patients with treatment-refractory Hodgkin lymphoma enrolled in the study had received a median of 6 prior therapies; 23 (82\%) had undergone autologous stem cell transplantation and $7(25 \%)$ allogeneic stem cell transplantation. Despite the poor responses to prior therapies, the overall response rate in the vorinostat/ sirolimus trial was 57\% (9/28 [32\%] CR and 7/28 [25\%] PR)[5] with tolerable toxic effects (dose interruptions only: 4/28 [25\%], dose interruptions and modifications: $15 / 28$ [54\%]). Considering that patients with refractory Hodgkin lymphoma generally have a poor clinical outcome, the combination of sirolimus and vorinostat appears to be a promising treatment strategy.

Interestingly, similar activity was shown by Oki et al in a trial of the combination of everolimus and panobinostat in patients with relapsed or refractory lymphoma. In 14 patients with Hodgkin lymphoma, the overall response rate was $43 \%(6 / 14)$ and the CR rate $14 \%(2 / 14)$ [16]. Notably, the discontinuation rate due to intolerance was higher $(43 \%$ [13/30]) than in our trial of sirolimus and vorinostat (4\% [1/28]). The lower rate in our study may have been related to, among other factors, more flexible dosing and dose adjustments for sirolimus and vorinostat, which are both FDA approved (although not for Hodgkin lymphoma). Finally, the sirolimus and vorinostat regimen has a relatively favorable cost compared to some other novel FDA-approved therapies such as brentuximab; the estimated cost for 1 cycle of brentuximab is $\$ 13,683$, while that for vorinostat/sirolimus is $\$ 7,696$. The cost of the vorinostat/sirolimus regimen is likely to drop even further when the vorinostat patent protection expires in the near future, making this combination attractive in settings of more limited resources. Nevertheless, so far advancing this combination further in the clinical trials trajectory has been difficult.

A final example is a phase I clinical trial of bevacizumab in combination with an EGFR- or HER2targeted agent. Preclinical data indicate that anti-VEGF treatment can augment response to HER2 or EGFR inhibition and increase apoptosis [17] and that dual blockade of tyrosine-kinase receptors with a monoclonal antibody at the extracellular domain and a tyrosine kinase inhibitor at the intracellular domain is effective [18]. These preclinical observations provided a rationale for combining anti-HER2 agents trastuzumab and lapatinib with bevacizumab in patients with metastatic HER2positive breast cancer pretreated with a median of 7 regimens, including HER2-targeted therapies. Patients were able to tolerate the recommended FDA-approved doses of all 3 agents. Of the 26 patients enrolled, 1 [4\%] had CR, 6 [23\%] had PR, and 6 [23\%] had SD $\geq 6$ months [3]. Similarly, among 34 patients with NSCLC refractory to a median of 4 prior therapies who were treated with bevacizumab, erlotinib, and cetuximab, 4 [12\%] had PR and 7 [21\%] SD $\geq 6$ months [4]. These combinations appear to have less favorable costs than other repurposing strategies mentioned; however, most of these compounds are nearing the end of their patent protection, and the costs are expected to drop significantly in the years to come. Unfortunately, we have been unable to secure adequate support to move these treatments along in the drug development pipeline.

\section{Clinical development challenges in drug repurposing strategies}

Historically, the expected response rate in Phase I trials with unselected populations of patients has ranged from $4 \%$ to $11 \%$ [19-22]. However, with recent advances in targeted therapies and molecular matching, the response rates in Phase I trials have ranged from $19 \%$ to $77 \%$, and some agents that demonstrated high response rates are now FDA approved [23-28]. Even in heavily pretreated patients, drug repurposing combination regimens have yielded response rates ranging from $12 \%$ to $57 \%$ [3-8] (Figure 1).

Despite these promising results and the lower costs of existing agents, financial support for drug repurposing approaches has been lacking. In fact, the lower drug prices, short patent duration, and low return on investment are likely reasons that the pharmaceutical industry is not interested in investing in such clinical trials. Therefore, we propose that such efforts be more easily funded through federal sources such as National Cancer Institute and other agencies [29].

\section{CONCLUSIONS}

Our experience has shown favorable outcomes with 
certain drug-repurposing strategies in cancer treatment, (Figure 1) which may reduce the cost and timeline for approval. However, this approach has several limitations. First, our studies were single institutional experience reviewing several investigator-initiated trials, nonrandomized, dose-finding trials in which some of the enrolled patients may have received less then optimal dose. Second, our studies enrolled relatively small numbers of patients, often with diverse treatment-refractory advanced malignancies, which may have complicated data interpretation. Third, a substantial proportion of the patients had already been treated with drugs included in the novel combinations.

Lack of funding and interest from the pharmaceutical industry limits the clinical development trajectory. Potential solutions include support from peerreviewed federal funding sources, which are centered more on patient and public health outcomes than commercial interests.

\section{CONFLICT OF INTEREST}

Dr. Kurzrock is a founder of RScueRX Inc.

\section{ACKNOWLEDGMENTS}

This manuscript was funded by the Sheikh Khalifa Al Nahyan Ben Zayed Institute for Personalized Cancer Therapy, by NCATS grant UL1 TR000371 (Center for Clinical and Translational Sciences), by the Jeanne F. Shelby Scholarship Fund, and by the MD Anderson Cancer Center Support grant (P30 CA016672).

\section{REFERENCES}

1. Group UCSW. United States cancer statistics: 1999-2006 incidence and mortality web-based report. Atlanta, GA. 2013.

2. Gupta SC, Sung B, Prasad S, Webb LJ and Aggarwal BB. Cancer drug discovery by repurposing: teaching new tricks to old dogs. Trends Pharmacol Sci. 2013; 34(9):508-517.

3. Falchook GS, Moulder SL, Wheler JJ, Jiang Y, Bastida CC and Kurzrock R. Dual HER2 inhibition in combination with anti-VEGF treatment is active in heavily pretreated HER2positive breast cancer. Ann Oncol. 2013; 24(12):3004-3011.

4. Falchook GS, Naing A, Hong DS, Zinner R, Fu S, Piha-Paul SA, Tsimberidou AM, Morgan-Linnell SK, Jiang Y, Bastida C, Wheler JJ and Kurzrock R. Dual EGFR inhibition in combination with anti-VEGF treatment: a phase I clinical trial in non-small cell lung cancer. Oncotarget. 2013; 4(1):118-127.

5. Janku F, Oki Y, Falchook GS, Subbiah V, Naing A, Velez Bravo VM, Hong DS, Westin JR, Nunez C, Fayad L, Neelapu SS, Kwak LW, Shpall EJ, Wheler JJ, Barnes TG,
Liang WS, et al. Activity of the mTOR inhibitor sirolimus and HDAC inhibitor vorinostat in heavily pretreated refractory Hodgkin lymphoma patients. American Society of Clinical Oncology Annual Meeting, Chicago, IL (abstract \#8508). 2014.

6. Moroney J, Fu S, Moulder S, Falchook G, Helgason T, Levenback C, Hong D, Naing A, Wheler J and Kurzrock R. Phase I study of the antiangiogenic antibody bevacizumab and the mTOR/hypoxia-inducible factor inhibitor temsirolimus combined with liposomal doxorubicin: tolerance and biological activity. Clin Cancer Res. 2012; 18(20):5796-5805.

7. Moroney JW, Schlumbrecht MP, Helgason T, Coleman RL, Moulder S, Naing A, Bodurka DC, Janku F, Hong DS and Kurzrock R. A phase I trial of liposomal doxorubicin, bevacizumab, and temsirolimus in patients with advanced gynecologic and breast malignancies. Clin Cancer Res. 2011; 17(21):6840-6846.

8. Moulder S, Moroney J, Helgason T, Wheler J, Booser D, Albarracin C, Morrow PK, Koenig K and Kurzrock R. Responses to liposomal Doxorubicin, bevacizumab, and temsirolimus in metaplastic carcinoma of the breast: biologic rationale and implications for stem-cell research in breast cancer. J Clin Oncol. 2011; 29(19):e572-575.

9. Pantuck AJ, Zeng G, Belldegrun AS and Figlin RA. Pathobiology, prognosis, and targeted therapy for renal cell carcinoma: exploiting the hypoxia-induced pathway. Clin Cancer Res. 2003; 9(13):4641-4652.

10. Rayson D, Adjei AA, Suman VJ, Wold LE and Ingle JN. Metaplastic breast cancer: prognosis and response to systemic therapy. Ann Oncol. 1999; 10(4):413-419.

11. Shaw AT, Yasothan U and Kirkpatrick P. Crizotinib. Nat Rev Drug Discov. 2011; 10(12):897-898.

12. Kirschbaum MH, Goldman BH, Zain JM, Cook JR, Rimsza LM, Forman SJ and Fisher RI. A phase 2 study of vorinostat for treatment of relapsed or refractory Hodgkin lymphoma: Southwest Oncology Group Study S0517. Leuk Lymphoma. 2012; 53(2):259-262.

13. Younes A, Oki Y, Bociek RG, Kuruvilla J, Fanale M, Neelapu S, Copeland A, Buglio D, Galal A, Besterman J, Li Z, Drouin M, Patterson T, Ward MR, Paulus JK, Ji Y, et al. Mocetinostat for relapsed classical Hodgkin's lymphoma: an open-label, single-arm, phase 2 trial. Lancet Oncol. 2011; 12(13):1222-1228.

14. Younes A, Sureda A, Ben-Yehuda D, Zinzani PL, Ong TC, Prince HM, Harrison SJ, Kirschbaum M, Johnston P, Gallagher J, Le Corre C, Shen A and Engert A. Panobinostat in patients with relapsed/refractory Hodgkin's lymphoma after autologous stem-cell transplantation: results of a phase II study. J Clin Oncol. 2012; 30(18):2197-2203.

15. Lemoine M, Derenzini E, Buglio D, Medeiros LJ, Davis RE, Zhang J, Ji Y and Younes A. The pan-deacetylase inhibitor panobinostat induces cell death and synergizes with everolimus in Hodgkin lymphoma cell lines. Blood. 
2012; 119(17):4017-4025.

16. Oki Y, Buglio D, Fanale M, Fayad L, Copeland A, Romaguera J, Kwak LW, Pro B, de Castro Faria S, Neelapu S, Fowler N, Hagemeister F, Zhang J, Zhou S, Feng L and Younes A. Phase I study of panobinostat plus everolimus in patients with relapsed or refractory lymphoma. Clin Cancer Res. 2013; 19(24):6882-6890.

17. Lichtenberger BM, Tan PK, Niederleithner H, Ferrara N, Petzelbauer P and Sibilia M. Autocrine VEGF signaling synergizes with EGFR in tumor cells to promote epithelial cancer development. Cell. 2010; 140(2):268-279.

18. Janku F, Huang HJ, Angelo LS and Kurzrock R. A kinaseindependent biological activity for insulin growth factor-1 receptor (IGF-1R) : implications for inhibition of the IGF1R signal. Oncotarget. 2013; 4(3):463-473.

19. Decoster G, Stein G and Holdener EE. Responses and toxic deaths in phase I clinical trials. Ann Oncol. 1990; 1(3):175181 .

20. Von Hoff DD and Turner J. Response rates, duration of response, and dose response effects in phase I studies of antineoplastics. Invest New Drugs. 1991; 9(1):115-122.

21. Horstmann E, McCabe MS, Grochow L, Yamamoto S, Rubinstein L, Budd T, Shoemaker D, Emanuel EJ and Grady C. Risks and benefits of phase 1 oncology trials, 1991 through 2002. N Engl J Med. 2005; 352(9):895-904.

22. Janku F, Garrido-Laguna I and Kurzrock R. Earlyphase Cancer Clinical Trials: Are the Goals Therapeutic or Scientific? American Society of Clinical Oncology, Educational Book. 2011.

23. Younes A, Bartlett NL, Leonard JP, Kennedy DA, Lynch $\mathrm{CM}$, Sievers EL and Forero-Torres A. Brentuximab vedotin (SGN-35) for relapsed CD30-positive lymphomas. N Engl J Med. 2010; 363(19):1812-1821.

24. Yap TA, Vidal L, Adam J, Stephens P, Spicer J, Shaw H, Ang J, Temple G, Bell S, Shahidi M, Uttenreuther-
Fischer M, Stopfer P, Futreal A, Calvert H, de Bono JS and Plummer R. Phase I trial of the irreversible EGFR and HER2 kinase inhibitor BIBW 2992 in patients with advanced solid tumors. J Clin Oncol. 2010; 28(25):39653972.

25. Von Hoff DD, LoRusso PM, Rudin CM, Reddy JC, Yauch RL, Tibes R, Weiss GJ, Borad MJ, Hann CL, Brahmer JR, Mackey HM, Lum BL, Darbonne WC, Marsters JC, Jr., de Sauvage FJ and Low JA. Inhibition of the hedgehog pathway in advanced basal-cell carcinoma. N Engl J Med. 2009; 361(12):1164-1172.

26. van Oosterom AT, Judson I, Verweij J, Stroobants S, Donato di Paola E, Dimitrijevic S, Martens M, Webb A, Sciot R, Van Glabbeke M, Silberman S, Nielsen OS, European Organisation for R, Treatment of Cancer Soft T and Bone Sarcoma G. Safety and efficacy of imatinib (STI571) in metastatic gastrointestinal stromal tumours: a phase I study. Lancet. 2001; 358(9291):1421-1423.

27. Fong PC, Boss DS, Yap TA, Tutt A, Wu P, MerguiRoelvink M, Mortimer P, Swaisland H, Lau A, O'Connor MJ, Ashworth A, Carmichael J, Kaye SB, Schellens JH and de Bono JS. Inhibition of poly(ADP-ribose) polymerase in tumors from BRCA mutation carriers. N Engl J Med. 2009; 361(2):123-134.

28. Flaherty KT, Puzanov I, Kim KB, Ribas A, McArthur GA, Sosman JA, O’Dwyer PJ, Lee RJ, Grippo JF, Nolop K and Chapman PB. Inhibition of mutated, activated BRAF in metastatic melanoma. N Engl J Med. 2010; 363(9):809-819.

29. Morello L. More cuts loom for US science. Nature. 2013; 501(7466):147-148.

30. Baselga J, Campone M, Piccart M, Burris HA, 3rd, Rugo HS, Sahmoud T, Noguchi S, Gnant M, Pritchard KI, Lebrun F, Beck JT, Ito Y, Yardley D, Deleu I, Perez A, Bachelot $\mathrm{T}$, et al. Everolimus in postmenopausal hormone-receptorpositive advanced breast cancer. N Engl J Med. 2012; 366(6):520-529. 